\title{
Aplicación de la lógica difusa en el ámbito de las energías renovables
}

\author{
Matilde Santos Peñas ${ }^{\star}$ Edurne Miranda Suescun ${ }^{\star \star}$ \\ Facultad de Informática, Universidad Complutense de Madrid, c/ Profesor García \\ Santesmaes s/n, 28040-Madrid, España
}

Fecha de entrega: Febrero 21 de 2012

FECHA DE EVAluación: Marzo 13 DE 2012

FECha DE APRobación: MAYo 3 DE 2012

\begin{abstract}
Resumen En este artículo se presenta la aplicación de una de las técnicas de Soft Computing, la lógica fuzzy o lógica difusa, al control de un sistema complejo y altamente no lineal como es un aerogenerador. Se describe el funcionamiento de estos sistemas eólicos, especificando las diferentes zonas de comportamiento que dan lugar a no linealidades difíciles de controlar por un solo regulador. Se han desarrollado los pasos necesarios para describir cómo se diseña y aplica un sistema basado en lógica difusa que se pueda aplicar a todas las zonas. Este controlador regula el ángulo de cabeceo y el par del aerogenerador. Se muestran resultados de simulación que validan la aplicabilidad de esta técnica inteligente para estos sistemas.
\end{abstract}

Palabras Clave: inteligencia artificial, lógica difusa, control fuzzy, aerogeneradores

\begin{abstract}
In this paper we present an application of Soft Computing techniques to a control problem. More specifically, we have applied fuzzy logic to control a wind turbine. These wind-driven generators are complex and highly non-linear systems. Firstly, the performance of these wind systems is described. They have different operating zones and therefore they are usually controlled by several controllers, one for each zone. The main goal of this paper is to develop an intelligent controller that works on every operating point. The design of a fuzzy controller for pitch angle and electric torque of a wind turbine is detailed. Simulation results are encouraging and show how this soft computing technique is suitable in order to control these types of complex systems.
\end{abstract}

Keywords: artificial intelligence, fuzzy logic, fuzzy control, wind turbines

\footnotetext{
* msantos@dacya.ucm.es

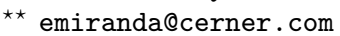




\section{Introducción}

El consumo de energía está muy relacionado con el crecimiento económico de una sociedad [7]. Históricamente, los combustibles fósiles como petróleo, carbono y gas natural han dominado el mercado de energía y todavía en el año 2009 contribuyeron con un $81 \%$ a la demanda energética mundial [2]. Sin embargo, el uso de los combustibles fósiles presenta una serie de complicaciones. Por un lado, la reproducción de los combustibles fósiles no tiene lugar a una velocidad significativa y, en consecuencia, pueden ser considerados como recursos finitos, con la repercusión que eso conlleva en el precio y el posible peligro de agotamiento de las reservas. Además, es importante hoy en día considerar el impacto ambiental derivado del uso de los combustibles fósiles, tanto a escala local como global.

Factores como éstos han contribuido al rápido crecimiento de las energías renovables en las últimas décadas $[11,2,7]$, y aparecen como una solución importante para el futuro. Las energías renovables ofrecen la posibilidad de generar electricidad y calor prácticamente sin emisiones, a bajos precios de operación y de manera sostenible. Además, lógicamente, los distintos países tienen mucho interés en aprovechar sus recursos renovables locales. Por ejemplo, España ha evolucionado como uno de los países líderes en las áreas de energía solar y eólica. España en el 2011 tenía una contribución eólica del $16 \%$ a la demanda anual, según el informe de la Red Eléctrica Española [6].

Las energías renovables tienen muchas ventajas, pero también presentan ciertos desafíos importantes. Una de las desventajas es la variabilidad de la fuente, sobre todo en el caso de la energía eólica. No es posible garantizar las condiciones ambientales con anticipación y, en consecuencia, tampoco el suministro. Además son sistemas difíciles de controlar debido a esta variabilidad.

Por otro lado, la inteligencia computacional está aportando soluciones a problemas reales hasta ahora no abordadas por su complejidad. En concreto, las técnicas de Soft Computing han sido aplicadas con éxito en numerosas aplicaciones, donde el control inteligente ha permitido solventar situaciones y sacar el máximo partido tanto recursos como instalaciones [10].

En esta investigación se muestra cómo se puede plantear la aplicación de la lógica difusa (fuzzy logic) al control de aerogeneradores, con el objetivo de hacer ver que esta técnica inteligente permite un mejor aprovechamiento de los recursos energéticos naturales.

Los aerogeneradores son sistemas complejos, no lineales, con funcionamiento dependiente de la zona de trabajo. Por ese motivo pensamos que la aproximación con lógica difusa es idónea. La motivación y objetivos del estudio son los siguientes:

- Actualmente la obtención de modelos fiables de aerogeneradores es complicada. Existen varios simuladores que permiten emular con cierta precisión aerogeneradores concretos, específicos. La mayoría de estos simuladores son capaces de calcular modelos lineales que son utilizados para el diseño de controladores, pero no dejan de ser modelos teóricos y, aunque pueden servir para el estudio de nuevas estructuras de control, normalmente los controladores diseñados con ellos no son directamente utilizables con las máquinas 
reales. Por ese motivo se han comenzado a realizar trabajos de identificación de modelos. En cualquier caso, la utilización de control difuso permitiría el diseño de un controlador sin necesidad de conocer la máquina en profundidad y sólo basado en las características de su curva de funcionamiento.

- Uno de los principales problemas de los controladores actuales es su funcionamiento en la zona de transición, ya que presentan al menos dos puntos de operación. Como los controladores convencionales están compuestos por dos lazos paralelos, la transición entre las dos zonas principales de funcionamiento del aerogenerador se arregla mediante saturaciones de uno u otro lazo, pero esta solución no permite una transición clara, y lleva asociada un aumento de las cargas en el aerogenerador. Es de esperar que si se utiliza un controlador difuso en el que se definen las reglas adecuadas que sirvan para todas las zonas de funcionamiento, el control en la zona de transición proporcione mejores resultados que los obtenidos con los controladores convencionales.

Por lo tanto el objetivo final de este trabajo de investigación es diseñar un sólo controlador difuso que funcione en todas las zonas de operación del aerogenerador.

El artículo está organizado como sigue. En la sección 2 se presentan los fundamentos del control basado en lógica fuzzy. En la sección 3 se describe el funcionamiento de un aerogenerador. En la sección 4 se detalla el diseño de un controlador difuso para un aerogenerador y se comentan los resultados de la simulación. El artículo termina con las conclusiones.

\section{Fundamentos del control basado en lógica difusa}

Una de las características esenciales de la lógica difusa es que permite utilizar el lenguaje ordinario como lenguaje de descripción en un ordenador, incorporando así el conocimiento de un experto en una tarea determinada. Introduce la imprecisión y la subjetividad propia de la actividad humana en un procedimiento automatizado. La lógica difusa emula una de las funcionalidades "inteligentes" de los seres humanos: el razonamiento con incertidumbre [9].

La lógica difusa parte de la redefinición del mismo pilar básico de las matemáticas: el concepto de conjunto [12]. La función de pertenencia generalizada de un elemento $x$ a un conjunto $A, \mu_{A}(x)$, no sólo valdrá 1 ó 0 , sino que toma sus valores en un conjunto $L$, llamado conjunto de pertenencia. Ordinariamente se toma el conjunto de números reales comprendidos entre 0 y 1 , inclusive: $L=[0,1]$.

La teoría clásica de conjuntos es un caso particular de la teoría de conjuntos difusos, en los que la función de pertenencia toma exclusivamente valores 0 ó 1 .

La función de pertenencia puede definirse de forma numérica, mediante un vector (para universos discretos y finitos), o de forma funcional, en cuyo caso tiene generalmente forma de campana, triangular, trapezoidal, etc. En este último caso además puede depender de algún parámetro que permita variar su forma, por ejemplo, de $\sigma$ en la función $\mu_{A}(x)$ descrita por la siguiente ecuación:

$$
\mu_{A}(x)=\exp \left(\frac{-\left(x-\mu_{A}(x)\right)^{2}}{2 \sigma_{A}^{2}}\right)
$$


Un conjunto difuso $A$ es un conjunto de pares ordenados de un elemento genérico $x$ que pertenece al universo de discurso $U$, y su grado de pertenencia al conjunto.

$$
A=\left\{x \mid \mu_{A}(x), \forall x \in U\right\}
$$

Las operaciones entre conjuntos difusos se pueden aplicar de forma análoga a como se definen para los conjuntos convencionales. Se pueden definir así operaciones como la complementación, la unión o la intersección. Además, a los conjuntos difusos se les pueden aplicar operadores o reglas de modificación, que corresponden a los modificadores lingüísticos (adverbios) que se utilizan con frecuencia en el lenguaje natural.

La lógica difusa tiene el mismo aspecto sintáctico que la lógica de predicados. Sin embargo, no constituye como ella un sistema axiomático puesto que no permite a partir de unos axiomas derivar otras sentencias válidas.

La semántica de la lógica difusa se basa en el concepto de vaguedad formalizado en la teoría de conjuntos difusos, pretende interpretar las sentencias, por ello, se debe hallar su significado o, lo que es lo mismo, calcular un subconjunto difuso que corresponda a la interpretación verdadera de tal sentencia. Los subconjuntos difusos que pueden interpretar el significado de una sentencia se denominan valores de verdad lingüísticos.

Las reglas difusas son reglas de producción, de la forma condicional si-entonces donde tanto los valores de las variables lingüísticas del antecedente como del consecuente son conjuntos difusos, es decir, resultados lingüísticos con una semántica asociada.

\subsection{Control difuso}

Tradicionalmente los sistemas de control inteligente combinan la teoría clásica de control con técnicas de inteligencia artificial [10]. Los sistemas de control inteligentes de mayor implantación son los controladores difusos. El objetivo de estos controladores es intentar compensar las discrepancias entre los valores observados y los esperados, utilizando ciertas reglas de actuación, especificadas mediante descriptores lingüísticos. Por ejemplo, la utilización de términos tales como muy caluroso o poco rápido representan información cualitativa poco precisa, pero son un elemento sustancial del conocimiento humano. Estos sistemas son fáciles de entender por el experto y su implantación es sencilla.

Un sistema de lógica difusa puro está formado únicamente por dos componentes: una base de reglas difusas del tipo si-entonces expresadas en forma lingüística, y un mecanismo de inferencias difusas que busca en la base de reglas las que son aplicables a la situación actual y opera con ellas de forma que el espacio de entradas sea proyectado en el espacio de salidas. Sin embargo, es habitual que las entradas y las salidas de los controladores sean números precisos. Para poder utilizar reglas difusas a partir de entradas y salidas concretas se suele utilizar un fuzzificador a la entrada y un defuzzificador a la salida. Así el sistema de lógica difusa presenta la estructura del esquema de la figura 1. La fuzzificación es el proceso mediante el cual se transforma un valor numérico concreto en una 


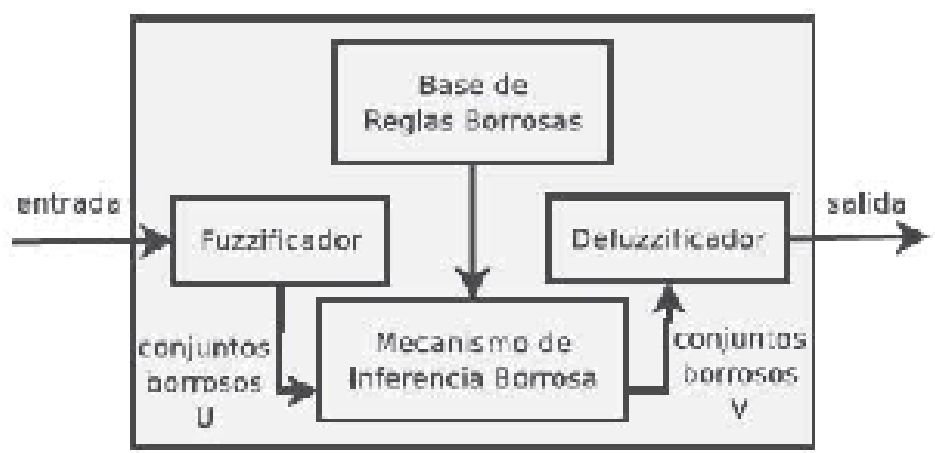

Figura 1. Sistema de lógica difusa.

variable de entrada en un conjunto difuso que representa dicho valor de forma imprecisa.

Si se desean utilizar reglas en un sistema de control, es necesario establecer qué términos lingüísticos van a ser utilizados. Para ello hay que dividir el universo de discurso o dominio en un número finito de conjuntos difusos, asociándoles una etiqueta lingüística a cada uno de ellos y asignándoles una función de pertenencia que los describa, para que puedan adoptar distintas formas.

La base de reglas difusas es la parte de la arquitectura del sistema de control difuso donde se almacena el conocimiento en forma de reglas. Un ejemplo de una de estas reglas sería:

\section{SI $x$ es A y $z$ es $\mathbf{C}$ ENTONCES $y$ es $\mathbf{F}$}

donde $\mathbf{A}, \mathbf{C}$ y $\mathbf{F}$ son conjuntos fuzzy. En cada instante discreto, el mecanismo de inferencias puede activar una, varias o ninguna de las reglas. La activación de una regla significa que un conjunto difuso de entradas definido sobre $U=U_{1} \times \cdots \times U_{n}$ (la entrada fuzzificada) es proyectado en un conjunto difuso de salida definido sobre $V$. Cada regla se activará en un grado distinto, dependiendo del grado en el que se satisfagan los antecedentes de la misma.

Durante el desarrollo de un sistema difuso, para establecer el mecanismo de inferencias de reglas, hay que seleccionar:

- Una interpretación de la implicación de las reglas si-entonces.

- Una regla de inferencia GMP (Modus Ponens Generalizado).

- Un operador de agregación para componer los resultados de las distintas reglas.

Una vez que la entrada ha sido procesada por el mecanismo de inferencias difusas, se obtiene una salida global, dada por un conjunto fuzzy. La defuzzificación se 
utiliza para elegir un valor de salida preciso (representativo), a partir de este conjunto difuso de salida.

\section{Descripción del funcionamiento de los aerogeneradores}

La estructura más habitual del aerogenerador hoy en día consiste en tres palas unidas a un buje (Figura 2). Todo este conjunto gira a una velocidad relativamente baja y dependiente del viento existente. Por este motivo se acopla el eje de giro del conjunto a una caja multiplicadora cuyo eje de salida (a velocidades más altas) hace girar el generador eléctrico que produce la energía, la cual posteriormente será vertida a la red [3]. Debido a la dependencia del funcionamiento del aerogenerador

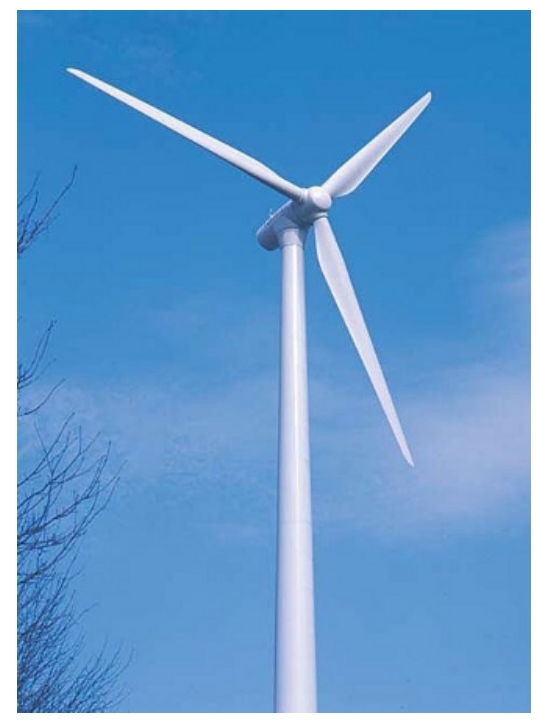

Figura 2. Aerogenerador de tres palas.

del viento existente se pueden distinguir diferentes zonas de funcionamiento, en cada una de las cuales el objetivo del sistema de control es diferente [1]. A vientos bajos, el objetivo consiste en maximizar la energía que se capta del viento; cuando el viento es suficientemente alto como para que el aerogenerador funcione a su potencia nominal, el objetivo de control debe ser mantener la máquina en dicha potencia. Entre ambas zonas de funcionamiento existe una zona de transición. La forma en la que el sistema de control actúa para obtener dichos objetivos es controlando la velocidad de giro.

Para explicar cómo se hace el control de velocidad, se puede considerar un modelo muy sencillo de aerogenerador en el que se suponen dos masas unidas 
mediante un eje. Por un lado tendríamos el rotor eólico (conjunto buje + palas) y por otro el generador eléctrico (Figura 3). El conjunto girará cuando se le aplique

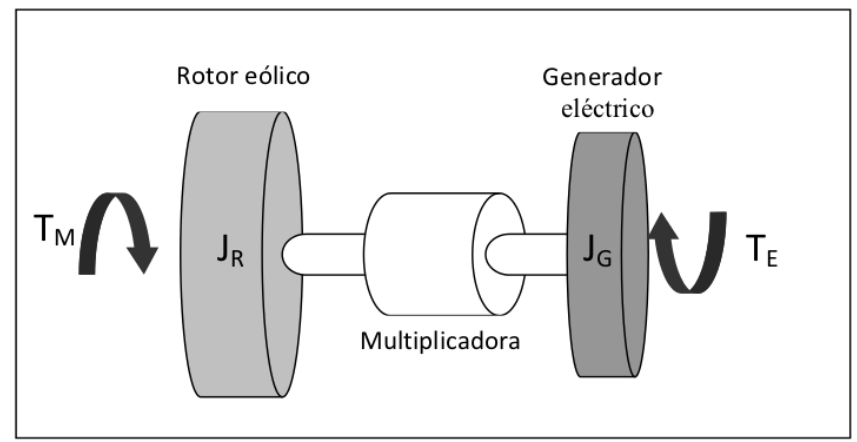

Figura 3. Aerogenerador modelado como sistema de dos masas.

un par motor (obtenido del viento) y se verá frenado por un par resistente (que corresponderá con el par eléctrico generado). La velocidad resultante dependerá de los momentos de inercia de ambas masas.

Por lo tanto, según lo expuesto hasta ahora, el control de la velocidad del aerogenerador se puede realizar mediante el control del par obtenido del viento o mediante el control del par eléctrico del aerogenerador. Para el control de la energía obtenida del viento, lo que se hace es controlar el ángulo con el que las palas lo reciben (ángulo de pitch o cabeceo). De esta forma se controla el viento efectivo que las mueve y por lo tanto la fuerza obtenida. El caso del control del par eléctrico es más directo porque dicha variable puede medirse y controlarse directamente.

A la determinación de la velocidad de giro mediante la modificación de estas variables se le conoce como control de velocidad [8]. Asociado a este control se desarrolla el control del generador eléctrico, que corresponde con el funcionamiento de la electrónica de potencia necesaria para que el generador responda a las demandas de par calculadas en el control de velocidad, pero este control queda fuera del ámbito de este trabajo. Por encima de estos controles existe un control supervisor que se encarga de dar las consignas adecuadas para el control de velocidad y de potencia, según la curva de funcionamiento del aerogenerador. Esta curva de funcionamiento determina la potencia eléctrica producida para las diferentes velocidades de viento a las que el aerogenerador puede trabajar. Dicha curva tiene la forma mostrada en la figura 4. Como se puede observar en la curva de potencia, el aerogenerador sólo produce potencia entre dos velocidades de viento determinadas, denominadas velocidad de conexión y velocidad de corte. Para velocidades inferiores a la de conexión, la energía del viento es demasiado 


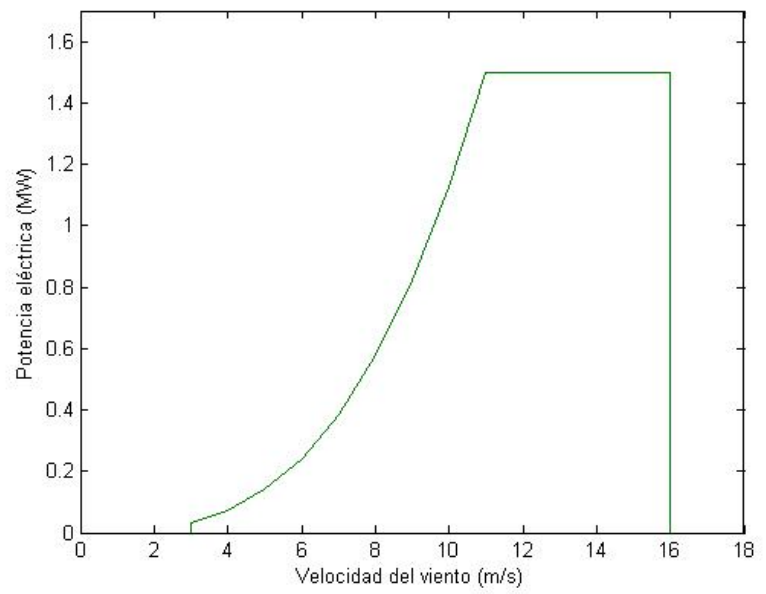

Figura 4. Potencia eléctrica vs velocidad del viento.

pequeña para producir potencia eléctrica, y para velocidades mayores que la velocidad de corte, podría ser peligroso para los componentes del aerogenerador.

Una vez explicado esto, se puede profundizar en el control de cada una de las zonas de funcionamiento del aerogenerador:

- Zona 1: cuando el viento es muy bajo se intenta mantener al aerogenerador girando a una velocidad menor que su velocidad nominal. En esta zona el control se realiza mediante la activación del lazo del par eléctrico mientras que el pitch es constante e igual a cierto valor de diseño.

- Zona 2: cuando la velocidad del viento es algo mayor pero no lo suficiente como para dar la energía correspondiente para producir la potencia nominal, lo que se intenta es obtener la máxima energía del viento posible. Esta potencia será mayor conforme aumenta la velocidad del viento ya que aumenta la energía del viento. Lo que se intenta optimizar es un parámetro que relaciona la velocidad de giro del rotor del aerogenerador con un valor de pitch óptimo. Por lo tanto para cada velocidad de viento (para cada punto de la curva de potencia) se pueden calcular fácilmente los valores de pitch y par eléctrico óptimos.

- Zona 3: cuando la potencia alcanza su valor nominal, ésta se debe mantener constante, por lo tanto ya no interesa obtener la máxima energía del viento sino mantener esta potencia y esa velocidad de giro constante. Para ello se varía el ángulo de pitch para desechar par motor y mantener la potencia nominal sin que el generador eléctrico se acelere.

Teniendo en cuenta que la velocidad del generador varía dependiendo de la zona de funcionamiento, se ha generado la figura 5 donde se muestra esta dependencia. 
Esto describe en general el funcionamiento del control de un aerogenerador

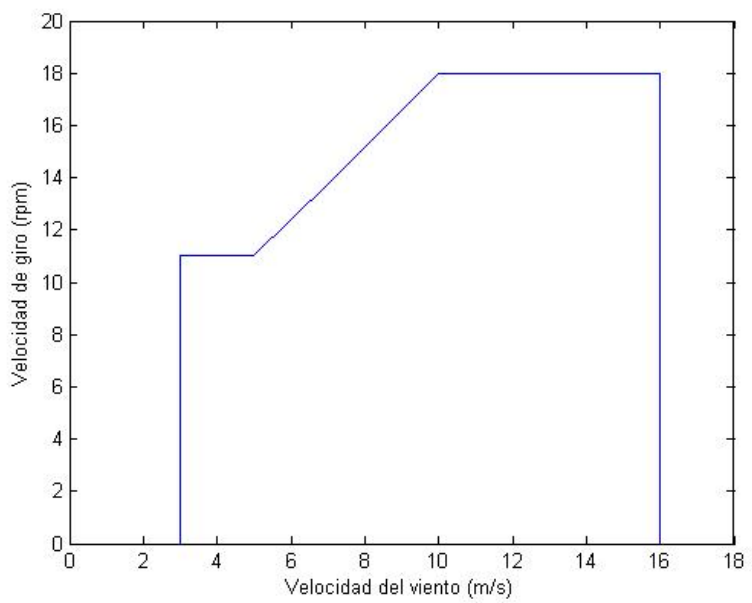

Figura 5. Velocidad de giro del rotor vs velocidad del viento.

aunque, hoy en día, ya que las máquinas son cada vez más grandes y están mejor diseñadas, se está restando importancia al objetivo de maximizar la potencia eléctrica obtenida, porque el control se está enfocando, en gran medida, a la disminución de las cargas a las que se ven sometidos los componentes mecánicos para poder aumentar su tiempo de vida. Con esa idea se van incorporando una serie de filtros ajustados a las diferentes frecuencias características del sistema.

\section{Diseño de un controlador basado en lógica difusa para un aerogenerador}

Como se ha dicho anteriormente, la estructura de control más habitual consiste en la utilización de dos lazos de control en paralelo. Ambos controles se encargan de controlar la velocidad de giro del generador pero en uno se utiliza como variable manipulada el ángulo de pitch y en el otro el par eléctrico del generador [5]. La activación de uno u otro lazo se realiza según la zona de funcionamiento en la que se encuentre el aerogenerador, de forma que sólo se activan cuando deben controlar la velocidad de giro y, en caso contrario, se saturan al valor correspondiente.

Por lo tanto se puede deducir que los modelos de aerogenerador necesarios para el diseño del control deben ser modelos multivariables con tres entradas (la velocidad del viento, el ángulo de pitch y el par del generador), y una salida, que 
corresponde con la velocidad del generador eléctrico. De estas tres entradas, el viento no es una entrada propiamente, ya que no se puede manipular, por lo que sería más correcto considerarlo como una perturbación.

Mientras que las variaciones del ángulo de pitch son lentas, debido al gran tamaño de las palas, las variaciones del par eléctrico en el generador son algo más rápidas. Hay que tener en cuenta que en el primer caso se actúa sobre un sistema mecánico y en el segundo sobre un sistema eléctrico, el cual siempre posee dinámicas más rápidas.

Hay otra circunstancia a tener en cuenta en estos modelos. Debido al giro de las palas, el par motor captado por las palas en su barrido contiene componentes periódicas cuyo periodo, en un aerogenerador tri-pala, corresponde con un tercio del periodo de giro de las palas. Esta periodicidad está causada principalmente por tres efectos:

- Por las componentes del viento no perpendiculares al plano de giro, pues éstas provocan que la velocidad relativa del viento sobre las palas dependa de su ángulo de azimut (el ángulo barrido en su giro).

- Por la presencia de la torre que crea perturbaciones en sus alrededores y que afectan, permanentemente sobre la energía captada del viento, cada vez que una de las palas pasa cerca de la torre.

- Y por último porque, habitualmente, el plano de giro de las palas no es completamente perpendicular al plano del suelo, lo que provoca desequilibrios periódicos debidos al peso de los diferentes elementos del aerogenerador.

Por último, hay que indicar que la ganancia estática de los modelos entre velocidad del generador y ángulo de pitch varía dependiendo de la velocidad de viento, por lo que normalmente, en los controladores convencionales, este lazo tiene una estrategia de control de ganancia programada.

En este trabajo se ha basado en el modelo de aerogenerador descrito en [4]. Tiene en cuenta las no linealidades del comportamiento de estos dispositivos.

\subsection{Estructura del regulador difuso}

Teniendo en cuenta lo anterior, se ha definido un regulador basado en lógica fuzzy, tanto para el lazo que corresponde al control del ángulo de cabeceo o pitch, como para el par.

Como entradas de control se consideran el error en la velocidad de giro del generador $\left(e_{w}\right)$ y en la potencia eléctrica $\left(e_{p o t}\right)$, así como sus derivadas $\left(d e_{w}, d e_{p o t}\right.$, respectivamente). El error se define como la diferencia entre el valor de referencia o salida deseada y el valor actual. Para trabajar con estas variables se han definido una serie de funciones de pertenencia mediante las cuales se intentan identificar las siguientes situaciones del cuadro 1. En las figuras 6 y 7 se muestran a modo de ejemplo las funciones de pertenencia asociadas a las variables error de potencia y derivada del error de velocidad del aerogenerador, respectivamente. Se han definido con forma triangular y están uniformemente distribuidas en los correspondientes universos del discurso. Como 


\begin{tabular}{ll}
\hline$e_{\text {pot }}>0$ & Por debajo de la potencia nominal \\
\hline$e_{p o t}=0$ & En potencia nominal \\
\hline$e_{\text {pot }}<0$ & Por encima de la potencia nominal \\
\hline$d e_{\text {pot }}>0$ & Nos alejamos de la potencia nominal \\
\hline$d e_{p o t}=0$ & Nos mantenemos igual respecto a la potencia nominal \\
\hline$d e_{p o t}<0$ & Nos acercamos a la potencia nominal \\
\hline$e_{w}>0$ & Por debajo de la velocidad nominal \\
\hline$e_{w}=0$ & En velocidad nominal \\
\hline$e_{w}<0$ & Por encima de la velocidad nominal \\
\hline$d e_{w}>0$ & Nos alejamos de la velocidad nominal \\
\hline$d e_{w}=0$ & Nos mantenemos respecto a la velocidad nominal \\
\hline$d e_{w}<0$ & Nos acercamos a la velocidad nominal \\
\hline
\end{tabular}

Cuadro 1. Zonas de trabajo del aerogenerador.

se puede observar, estas funciones de pertenencia están enfocadas al control en la zona de par nominal. Para el funcionamiento en el resto de zonas es necesario definir más funciones de pertenencia que permitan conocer no sólo si se está por encima o debajo, acercándonos o alejándonos de las condiciones nominales, sino también cuánto alejados o cercanos estamos. Como el objetivo de este trabajo es mostrar la viabilidad de una de las técnicas de soft computing en una aplicación real, se ha mantenido el problema en términos simples para no oscurecer su comprensión.

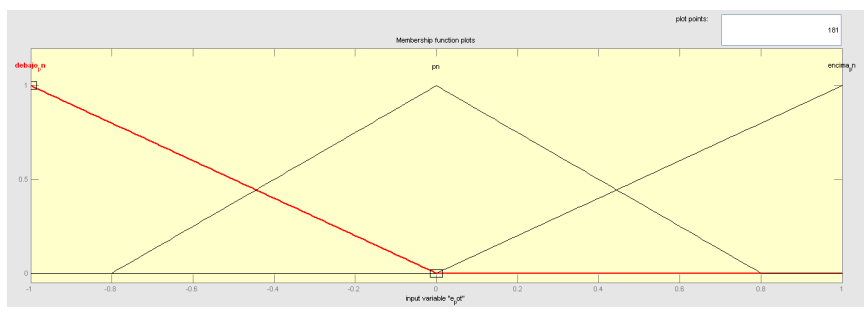

Figura 6. Funciones de pertenencia para la variable de entrada "error de potencia".

Las variables de salida con las que se está trabajando son el ángulo de pitch y el par del generador, para las cuales se han definido tres funciones de pertenencia como se muestra a continuación en la figura 8 tanto para el par de potencia, como para el ángulo de cabeceo.

Tanto el modelo del aerogenerador como los controladores están diseñados en Matlab /Simulink, en concreto se ha utilizado la herramienta Fuzzy de Matlab. Una vez definidas las variables, se determinan las reglas del controlador difuso 


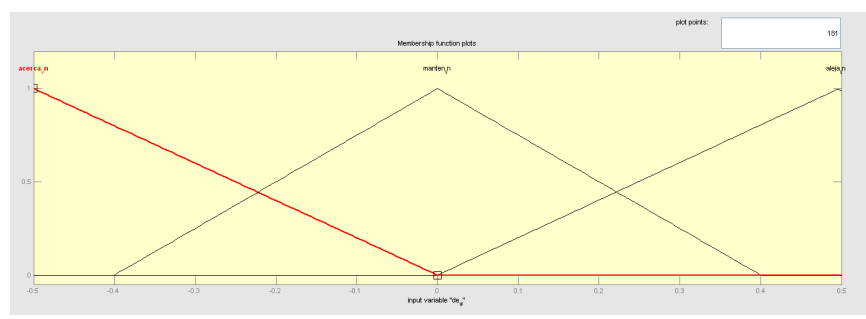

Figura 7. Funciones de pertenencia para la variable de entrada "derivada del error de velocidad".

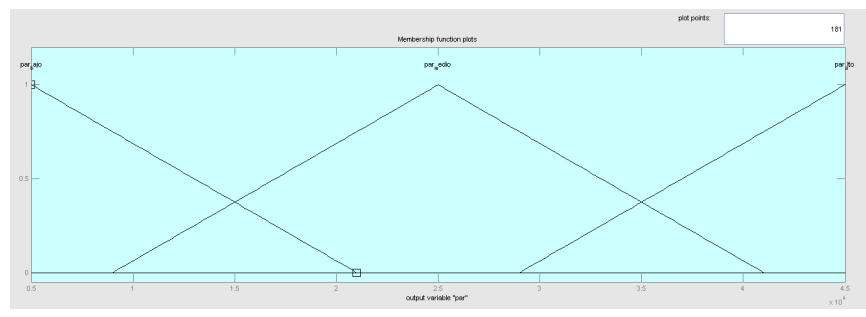

Figura 8. Funciones de pertenencia para la variable de salida par.

que inferirán la acción de control, en función de los valores de las entradas. Las reglas son del tipo:

IF $x$ is $\mathbf{A}$ and $y$ is $\mathbf{B}$ THEN $z$ is $\mathbf{C}$

donde A, B, y $\mathbf{C}$ son conjuntos difusos. En nuestro caso, se han definido realmente dos controladores fuzzy, cada uno de los cuales tienen dos entradas y una salida. Por lo tanto, las reglas son como se muestran en la figura 9. Estas reglas corresponden a la acción de controladores del tipo fuzzy- $P D$, con acción proporcional y derivativa. La superficie de control generada para cada uno de ellos es la presentada en la figura 10. Como se puede observar, su comportamiento es claramente no lineal. La configuración del sistema depende del diseñador. En este caso se ha implementado la conectiva "and" como el mínimo, y se ha aplicado el centro del área como método de defuzzificación.

En la figura 11 se muestra el resultado del controlador de potencia. Como se pueda observar, las fluctuaciones del viento influyen bastante en la estabilidad de la salida.

No se han obtenido muy buenos resultados debido a la complejidad del modelo y porque no existe un método sistemático para la sintonía de los controladores difusos. Sin embargo, el controlador sí consigue mantener la salida en torno a la referencia, a pesar de la variabilidad de las entradas. 
1. If (e_pot is debajo_pn) and (de_pot is aleja_pn) then (par is par_alto) (1)

2. If (e_pot is debajo_pn) and (de _pot is manten_pn) then (par is par_alto) (1)

3. If (e_pot is debajo_pn) and (de_pot is aleja_pn) then (par is par_medio) (1)

4. If (e_pot is pn) and (de_pot is aleja_pn) then (par is par_alto) (1)

5. If (e_pot is pn) and (de_pot is manten_pn) then (par is par_medio) (1)

6. If (e_pot is pn) and (de_pot is acerca_pn) then (par is par_bajo) (1)

7. If (e_pot is encima_pn) and (de_pot is aleja_pn) then (par is par_medio) (1)

8. If (e_pot is encima_pn) and (de_pot is manten_pn) then (par is par_bajo) (1)

9. If (e_pot is encima_pn) and (de_pot is acerca_pn) then (par is par_bajo) (1)

10. If (e_w is debajo_wn) and (de_w is aleja_vn) then (pitch is pitch_grande) (1)

11. If (e_w is debajo_wn) and (de_w is manten_wn) then (pitch is pitch_grande) (1)

12. If ( $e_{-} w$ is debajo_wn) and (de_w is acerca_wn) then (pitch is pitch_mediano) (1)

13. If (e_w is $w n$ ) and (de_w is aleja_vn) then (pitch is pitch_grande) (1)

14. If (e_w is $w n$ ) and (de_w is manten_vn) then (pitch is pitch_mediano) (1)

15. If (e_w is $w n$ ) and (de_w is acerca_vn) then (pitch is pitch_pequeño) (1)

16. If (e_w is encima_vn) and (de_w is aleja_vn) then (pitch is pitch_mediano) (1)

17. If (e_w is encima_vn) and (de_w is manten_vn) then (pitch is pitch_pequeño) (1)

18. If (e_w is encima_vn) and (de_w is acerca_vn) then (pitch is pitch_pequeño) (1)

Figura 9. Conjunto de reglas difusas de los controladores del aerogenerador.

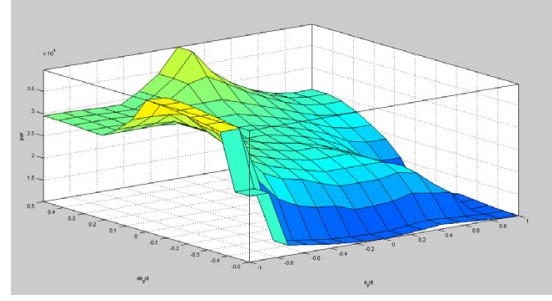

(a)

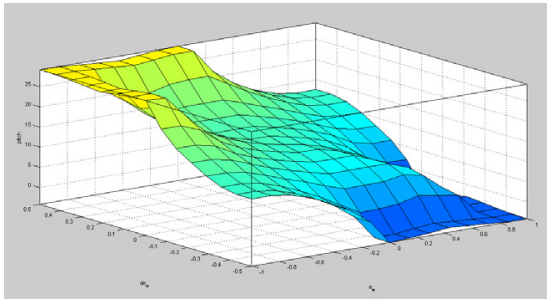

(b)

Figura 10. Superficie de control del sistema difuso para el controlador del par (a) y del ángulo de cabeceo (b).

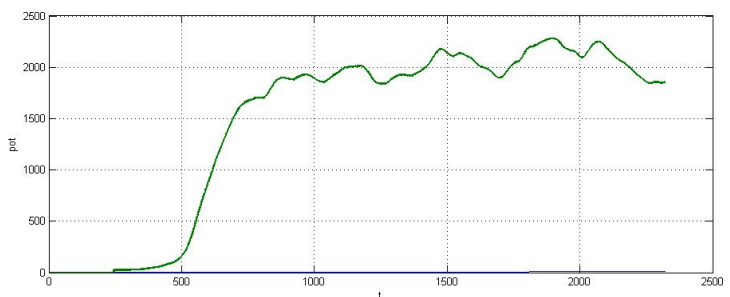

Figura 11. Salida del controlador difuso de potencia del aerogenerador. 


\section{Conclusiones}

Se ha demostrado cómo la aplicación de una de las técnicas del soft computing, en concreto la lógica fuzzy, permite abordar problemas complejos como es el control de sistemas altamente no lineales. En este caso, se ha aplicado a un aerogenerador.

Se ha mostrado cómo se tratan las variables de este sistema de forma difusa, estableciendo conjuntos fuzzy dentro de su rango de variación y definiendo unas reglas que permitan inferir la salida del sistema. En concreto se han diseñado dos controladores difusos, uno para el ángulo de cabeceo y otro para el par de un aerogenerador de tres palas.

\section{Agradecimientos}

Este trabajo ha sido parcialmente financiado por el Proyecto CICYT DPI2009 14552 - C02 - 01 del Ministerio de Ciencia e Innovación de España.

\section{Referencias}

1. Burton, T., Sharpe, D., Jenkins N., Bossanyi E.: Wind energy handbook. John Wiley \& Sons (2001)

2. IEA International Energy Agency. Key World Energy Statistics. http://www.iea. org (2011)

3. Khan, M. J., Iqbal, M.T.: Analysis of a small wind-hydrogen stand-alone hybrid energy system. Applied Energy 86, 2429-2442 (2009)

4. Mikati, M., Santos, M., Armenta, C.: Modelado y Simulación de un Sistema Conjunto de Energía Solar y Eólica para Analizar su Dependencia de la Red Eléctrica, RIAI, in press (2012)

5. Muljadi E., Butterfield C.P.: Pitch-Controlled Variable-Speed Wind Turbine Generation, IEEE Trans. on Industrial Applications, 37, 1, 240-246 (2001)

6. REE, Red eléctrica de España. Avance del informe. http://www.ree.es/ (2011).

7. REN21 Renewable Energy Network for the $21^{\text {th }}$ Century. Renewable Global Status Report. http://www.ren21.net/ (2011)

8. González, M., Vázquez F., Morilla F.: Control Multivariable Centralizado con Desacoplo para Aerogeneradores de Velocidad Variable. RIAI 7(4), 53-64 (2010)

9. Santos, M.: Lógica fuzzy, aproximación al razonamiento humano. Cooperación Internacional, 7, 93-112 (2001).

10. Santos M.: Un enfoque aplicado del control inteligente, Revista Iberoamericana de Automática e Informática Industrial, 8, 283-296 (2011)

11. Twidell J., Weir T.: Renewable Energy Resources. Taylor \& Francis (2006)

12. Zadeh, L.A.: Fuzzy sets. Information and Control, 8, 338-353 (1965) 